\title{
Exoskeletal Force-Sensing End-Effectors With Embedded Optical Fiber-Bragg-Grating Sensors
}

\author{
Yong-Lae Park, Student Member, IEEE, Seok Chang Ryu, Richard J. Black, Member, IEEE, \\ Kelvin K. Chau, Member, IEEE, Behzad Moslehi, Member, IEEE, and Mark R. Cutkosky, Member, IEEE
}

\begin{abstract}
Force sensing is an essential requirement for dexterous robot manipulation. We describe composite robot end-effectors that incorporate optical fibers for accurate force sensing and estimation of contact locations. The design is inspired by the sensors in arthropod exoskeletons that allow them to detect contacts and loads on their limbs. In this paper, we present a fabrication process that allows us to create hollow multimaterial structures with embedded fibers and the results of experiments to characterize the sensors and controlling contact forces in a system involving an industrial robot and a two-fingered dexterous hand. We also briefly describe the optical-interrogation method used to measure multiple sensors along a single fiber at kilohertz rates for closed-loop force control.
\end{abstract}

Index Terms-Biologically inspired robots, dexterous manipulation, fiber Bragg grating (FBG), force and tactile sensing, force control, shape-deposition manufacturing.

\section{INTRODUCTION}

$\mathbf{F}$ UTURE robots are expected to free human operators from difficult and dangerous tasks that require dexterity in various environments. Prototypes of these robots already exist for applications such as extravehicular repair of manned spacecraft and robotic surgery, in which accurate manipulation is crucial. Ultimately, we envision robots operating tools with levels of sensitivity, precision, and responsiveness to unexpected contacts that exceed the capabilities of humans, thus making use of numerous force and contact sensors on their arms and fingers.

However, compared with even the simplest of animals, today's robots are lacking in terms of their sensing abilities. For example, a spider has as many as 325 mechanoreceptors on each leg [18], in addition to hair sensors and chemical sensors [3], [52]. Mechanoreceptors such as the slit sensilla of spiders [4], [8] and campaniform sensilla of insects [37], [53]

Manuscript received May 23, 2009; revised September 15, 2009. First published October 30, 2009; current version published December 8, 2009. This paper was recommended for publication by Associate Editor A. Albu-Schäffer and Editor K. Lynch upon evaluation of the reviewers' comments. This paper was presented in part at the 2007 IEEE International Conference on Robotics and Automation, Rome, Italy, and in part at the 2008 IEEE International Conference on Robotics and Automation, Pasadena, CA. This work was supported by the National Aeronautics and Space Administration under Small Business Innovation Research Contract NNJ06JA36C.

Y.-L. Park, S. C. Ryu, and M. R. Cutkosky are with the Center for Design Research, Stanford University, Stanford, CA 94305 USA (e-mail: ylpark@ stanford.edu; scryu@stanford.edu; cutkosky@ stanford.edu).

R. J. Black and B. Moslehi are with Intelligent Fiber Optic Systems Corporation, Santa Clara, CA 95054 USA (e-mail: rjb@ifos.com; bm@ifos.com).

K. K. Chau was with Intelligent Fiber Optic Systems Corporation, Santa Clara, CA 95054 USA. He is now with Glimmerglass Networks, Hayward, CA 94545 USA (e-mail: chau@ glimmerglass.com).

Color versions of one or more of the figures in this paper are available online at http://ieeexplore.ieee.org.

Digital Object Identifier 10.1109/TRO.2009.2032965 are especially concentrated near the joints, where they provide information about loads imposed on the limbs - whether due to regular activity or unexpected events such as collisions. By contrast, robots generally have a modest number of sensors that are often associated with actuators or concentrated in devices such as a force-sensing wrist. (For example, the Robonaut humanoid robot has 42 sensors in its hand and wrist module [9].) As a result, robots often respond poorly to unexpected and arbitrarily located impacts. The work in this paper is a part of a broader effort aimed at creating light-weight, rugged appendages for robots that, like the exoskeleton of an insect, feature embedded sensors so that the robot can be more aware of both anticipated and unanticipated loads in real time.

Part of the reason for the sparseness of force and touch sensing in robotics is that traditional metal and semiconductor strain gages are tedious to install and wire. The wires are often a source of failure at joints, and are the receivers for electromagnetic noise. The limitations are, particularly, severe for force and tactile sensors on the fingers of a hand. Various groups have explored optical fibers for tactile sensing, where the robustness of the optical fibers, the immunity to electromagnetic noise, and the ability to process information with a charge-coupled device (CCD) or complementary metal-oxide-semiconductor camera are advantageous [12], [26], [34]. Optical fibers have also been used to measure bending in the fingers of a glove [24] or other flexible structures [11], where the light loss is a function of the curvature. In addition, a single fiber can provide a highbandwidth pathway to take tactile and force information down the robot arm [2].

We focus on a particular class of optical sensors, i.e., fiber Bragg grating (FBG) sensors, which are finding increasing applications in structural health monitoring [1], [29], [30], and other specialized applications in biomechanics [10], [13] and robotics [42], [44]. FBG sensors have been attached to or embedded in the metal parts [17], [31] and composites [55] to monitor forces, strains, and temperature changes. FBG sensors are particularly attractive for applications where immunity to electromagnetic noise, small size, and resistance to harsh environments are important. Examples include space or underwater robots [16], [19], [56], medical devices (especially for use in MRI fields) [43], [63], and force sensing on industrial robots with large motors operating under pulsewidth-modulated control [17], [64].

FBG sensors reflect light with a peak wavelength that shifts in proportion to the strain to which they are subjected. The sensitivity of regular FBGs to axial strain is approximately $1.2 \mathrm{pm} / \mu \varepsilon$ at $1550 \mathrm{~nm}$ center wavelength [7], [28]. With the appropriate 


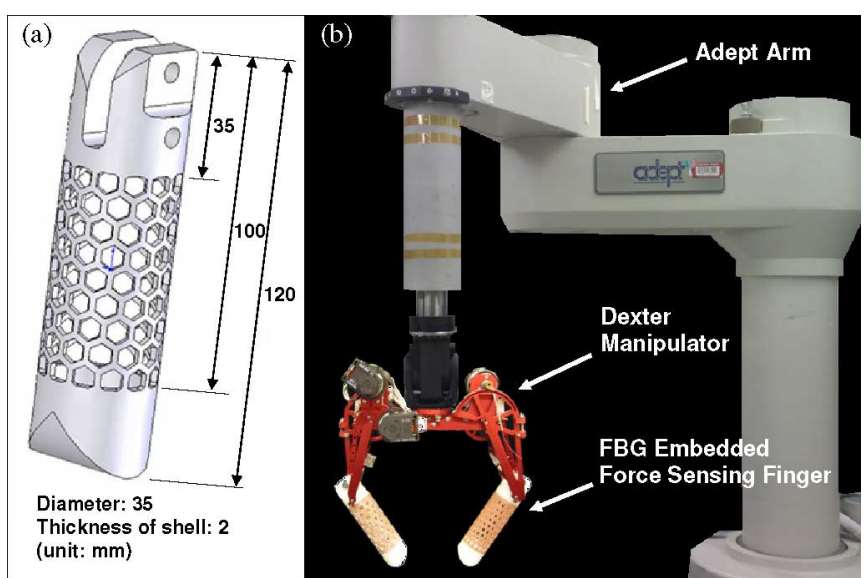

Fig. 1. (a) Prototype dimensions. (b) FBG-embedded force-sensing finger prototypes integrated with two-fingered hand and industrial robot.

FBG interrogator, very small strains, on the order of $0.1 \mu \varepsilon$, can be measured. In comparison to conventional strain gages, this sensitivity allows FBG sensors to be used in sturdy structures that experience modest stresses and strains under normal loading conditions. The strain response of FBGs is linear with no indication of hysteresis at temperatures up to $370^{\circ} \mathrm{C}$ [38] and, with appropriate processing, as high as $650^{\circ} \mathrm{C}$ [41]. Multiple FBG sensors can be placed along a single fiber and optically multiplexed at kilohertz rates.

To the best of our knowledge, the study in this paper is the first application of FBG sensors in hollow, bioinspired multimaterial robot limbs. The rest of this paper is organized as follows. Section II discusses the design concepts for the forcesensing finger prototype. Section III describes the fabrication process using a new variation of a rapid prototyping process. Section IV addresses the static and the dynamic characterization of the sensorized finger structures, including the ability to localize contact forces. Sections V and VI describe the hand controller used with the finger and the results of force control experiments. In Section VII, we present the results of our ongoing work to miniaturize the technology so that multiple FBG sensors can be applied to human-scale robotic fingertips or tools. In Section VIII, we discuss the optical-interrogation technology to read the strains from multiple sensors at sufficient rates for closed-loop force control. We conclude with a discussion of future work, which includes a potential extension of the finger prototype with a larger number of sensors for the measurement of external forces and contact locations. Future work also includes extending the capability of the optical interrogator and using multicore polymer fibers.

\section{DESIGN CONCEPTS}

Prototype fingers were designed as replacements for aluminum fingers on a two-fingered dexterous hand used with an industrial robot for experiments on force control and tactile sensing [20], as shown in Fig. 1. Fig. 2 shows a completed finger prototype including cross-sectional views. Each of the two fingers can be divided into three parts: fingertip, shell, and joint.
The fingertip and shell are exoskeletal structures. Four FBG sensors are embedded in the shell for strain measurement, and one FBG sensor is placed at the center of the finger for temperature compensation. The remainder of this section describes the design features of the prototype including the exoskeleton structure, solutions to reduce creep, and the effects of temperature variations and sensor placement.

\section{A. Exoskeleton Structure}

In comparison to solid structures, exoskeletal structures have high specific stiffness and strength. In addition, unlike a solid beam, they exhibit distinct local, as well as global, responses to contact forces (see Fig. 3). This property facilitates the estimation of contact locations. The exoskeletal structure may be compared with the plastic fingertip described by Voyles et al. [57], which used electrorheological fluids and capacitive elements for extrinsic tactile sensing, and required an additional cantilever beam with strain gages for force-torque information.

To enhance the deformation in response to local contact forces, our exoskeleton is designed as a grid. Although a grid structure with embedded FBG sensors has been explored for structural health monitoring on a large scale [1], it has rarely been considered in robotics. The ribs of the grid are thick enough to encapsulate the optical fibers and undergo axial and bending strains as the grid deforms. Although various polygonal patterns including triangles and squares are possible, hexagons have the advantage of minimizing the ratio of perimeter to area [21], [45], thereby reducing the weight of the part. Also, the hexagonal pattern avoids sharp interior corners, which could reduce the fatigue life. The thickness of the shell and the width of the pattern were determined so that each finger can withstand normal loads of at least $12 \mathrm{~N}$.

\section{B. Creep Prevention and Thermal Shielding}

Polymer structures experience greater creep than metal structures. Creep adversely affects the linearity and repeatability of the sensor output. In addition, thermal changes will affect the FBG signals. Drawing inspiration from a polymer hand by Dollar et al. [15], a copper mesh (080X080C0055W36T, TWP, Inc., Berkeley, CA) was embedded into the shell to reduce creep and provide some thermal shielding for the optical fibers. The high thermal conductivity of copper expedites the distribution of heat applied from outside the shell and creates a more uniform temperature within.

\section{Strain-Sensor Configuration}

In general, larger numbers of sensors will provide more information, and make the system more accurate and reliable. However, since additional sensors increase the cost and require more time and/or processing capacity, the optimal sensor configuration should be considered, as discussed by Bicchi and Canepa [5]. In the present case, if we assume that we have a single point of contact, then there are five unknown values: the longitude and latitude of a contact on the finger surface and the three orthogonal components of the contact force vector in the 

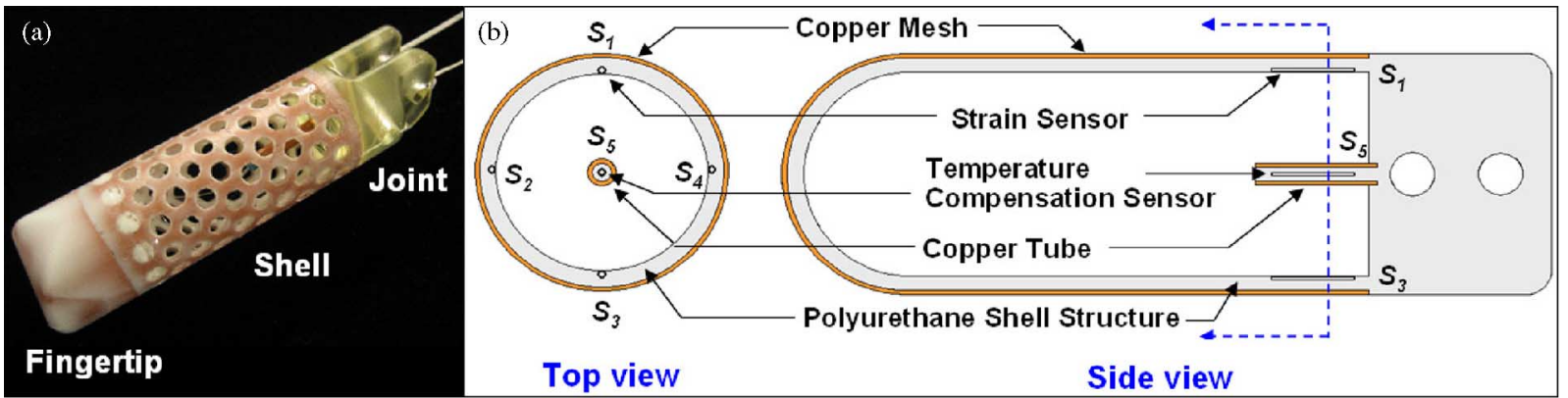

Fig. 2. (a) Finger prototype. (b) Cross-sectional views $\left(S_{1}-S_{4}\right.$ : strain sensors, $S_{5}$ : temperature-compensation sensor). See Table I for sensor parameters.

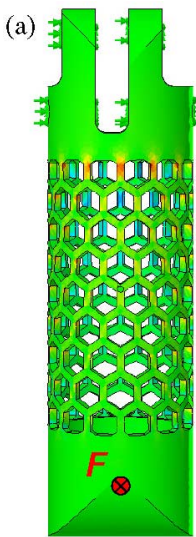

Front View

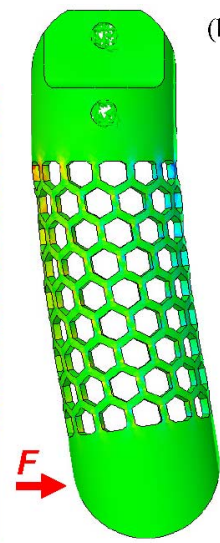

Right View

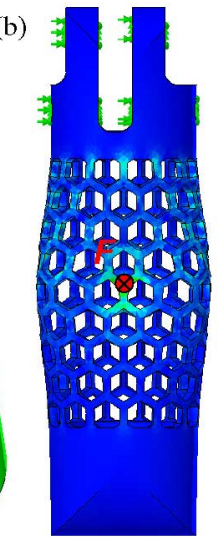

Front View

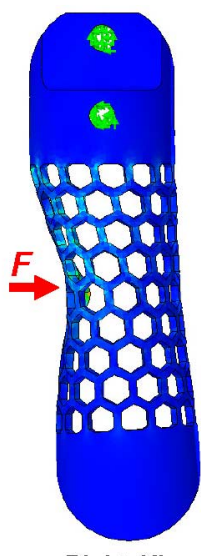

Right View
Fig. 3. Finite-element models showing strain concentrations on the first rib closest to the fixed joint. (a) Point load is applied to the fingertip. (b) Point load is applied to the middle of the shell structure.

$X, Y$, and $Z$ directions. For the initial finger prototypes, we further simplify the problem by assuming that the contact force is normal to the finger surface (i.e., with negligible friction). This assumption reduces the number of unknowns to three so that a minimum of three independent sensors are needed. In the prototype, four strain sensors were embedded in the shell.

Before fabrication, finite-element analysis was conducted to determine the sensor locations. Fig. 3 shows strain distributions when different types of forces are applied to the shell and fingertip. Strain is concentrated at the top of the shell, where it is connected to the joint. The four sensors were embedded at $90^{\circ}$ intervals into the first rib of the shell, which is closest to the joint, as shown in Fig. 2.

\section{Temperature Compensation}

Since embedded FBG sensors are sensitive to temperature, it is necessary to isolate thermal effects from mechanical strains. The sensitivity of regular FBGs to temperature change is approximately $10 \mathrm{pm} /{ }^{\circ} \mathrm{C}$ at $1550 \mathrm{~nm}$ center wavelength [22], [25]. Various complicated temperature-compensation methods have been proposed, such as the use of dual-wavelength superimposed FBG sensors [60], saturated chirped FBG sensors [61], and an FBG sensor rosette [35]. We chose a simpler method
TABLE I

PARAMETERS OF EMBEDDED FBG SENSORS

\begin{tabular}{cccc}
\hline Sensor & Wavelength & Bandwidth & Reflectivity \\
\hline$S_{1}$ & $1543.490 \mathrm{~nm}$ & $0.380 \mathrm{~nm}$ & $99.55 \%$ \\
$S_{2}$ & $1545.207 \mathrm{~nm}$ & $0.360 \mathrm{~nm}$ & $99.34 \%$ \\
$S_{3}$ & $1547.859 \mathrm{~nm}$ & $0.370 \mathrm{~nm}$ & $98.25 \%$ \\
$S_{4}$ & $1549.925 \mathrm{~nm}$ & $0.310 \mathrm{~nm}$ & $97.70 \%$ \\
$S_{5}$ & $1553.100 \mathrm{~nm}$ & $0.400 \mathrm{~nm}$ & $99.58 \%$ \\
\hline
\end{tabular}

that involved the use of an isolated, strain-free FBG sensor to measure thermal effects. Subtracting the wavelength shift of this sensor from that of any other sensor corrects for the thermal effects on the latter [47]. An important assumption in this method is that all the sensors experience the same temperature. Our prototype has one temperature compensation sensor in the hollow area inside the shell, as shown in Fig. 2. Although it is distanced from the strain sensors, the previously mentioned copper heat shield results in an approximately uniform temperature within the shell. Since the temperature compensation sensor is encapsulated in a copper tube attached at one end to the joint, it experiences no mechanical strain.

\section{Shape Deposition MANufacturing FABRICATION PROCEDURE}

The finger prototype was fabricated using a variation of the shape deposition manufacturing (SDM) rapid-prototyping process [58] to make a hollow 3-D part. The prototype was cast in a three-step process, shown in Fig. 4, with no direct machining required.

The base material is polyurethane, which is chosen for its combination of fracture toughness, ease of casting at room temperature, and minimal shrinkage. In particular, the urethane has a low mixed viscosity $(150 \mathrm{~Hz})$, which helps it to completely fill the narrow channels associated with ribs in the grid structure.

The first step is to cast the shell (see step 1 (a)-(d) in Fig. 4). The outer mold is made of hard wax to maintain the overall shape. The inner mold is hollow and made of silicone rubber, which can be manually deformed and removed when the polyurethane is cured. The optical fibers and copper mesh were embedded in this step. Although it is often preferable to strip the $50-\mu \mathrm{m}$ polyimide coating on FBG regions before optical fibers are embedded, we found that adequate bonding was obtained between the polyurethane and the coated fibers, and the amount 

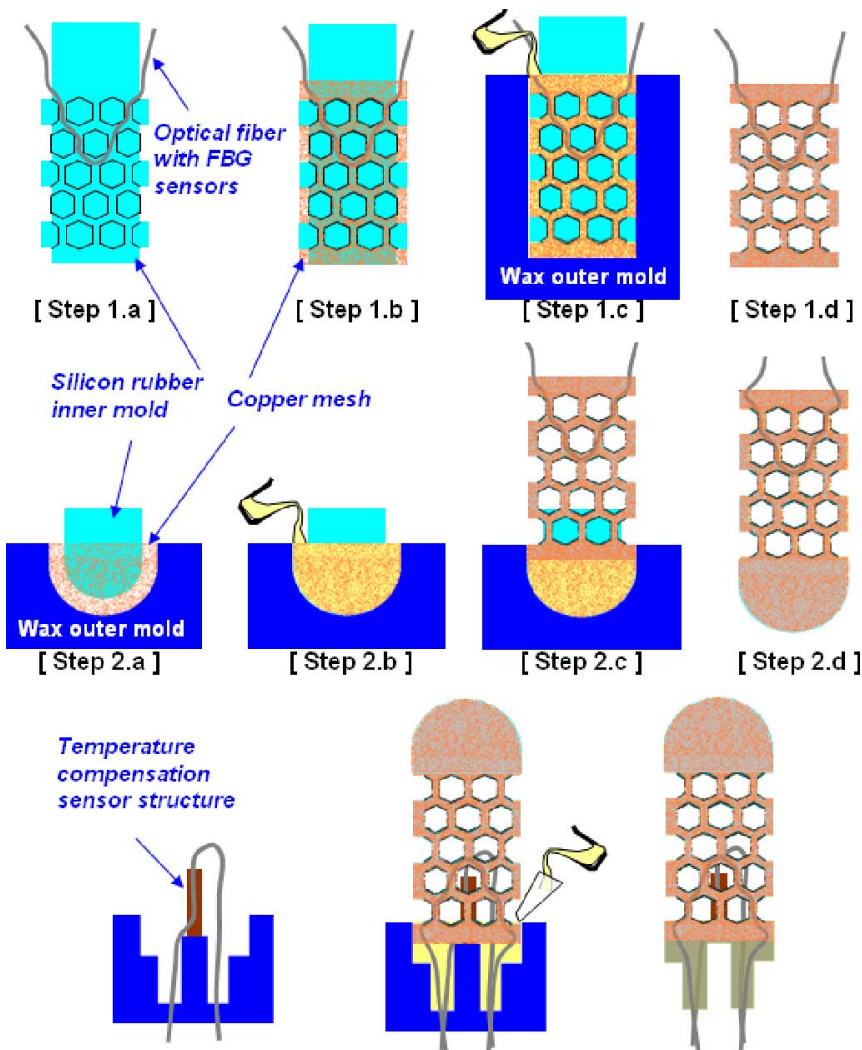

[Step 3.a ]

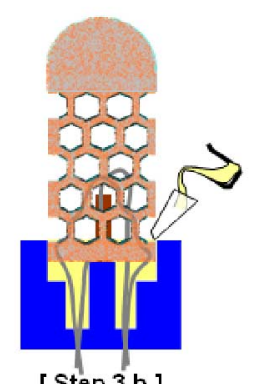

[ Step 3.b ]
[Step 1.d ]

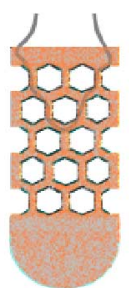

[ Step 2.d ]

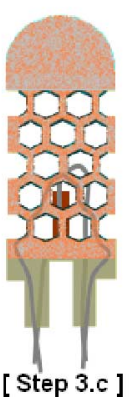

Fig. 4. Modified SDM fabrication process. (Step 1) Shell fabrication. (a) Prepare silicone rubber inner mold and place optical fibers with FBG sensors. (b) Wrap the inner mold with copper mesh. (c) Enclose inner mold and copper mesh with a wax outer mold and pour liquid polyurethane. (d) Remove inner and outer molds when polyurethane cures. (Step 2) Fingertip fabrication. (a) Prepare inner and outer molds and place copper mesh. (b) Cast liquid polyurethane (c) Place cured shell into the uncured polyurethane. (d) Remove molds when the polyurethane cures. (Step 3) Joint fabrication. (a) Prepare outer mold and place temperature compensation sensor structure. (b) Place cured shell and fingertip into the uncured polyurethane. (c) Remove outer mold when polyurethane cures.

of creep was negligible compared with overall deformation and creep in the urethane structure. Retaining the coating also protected the fibers during the casting process.

The second step is fingertip casting [see step 2 (a)-(d)], which uses separate molds and occurs after the shell is cured. The polyurethane for the fingertip bonds to the cured shell part.

In the final step, the joint is created [see step 3 (a)-(c)]. As with the fingertip, the joint bonds to the cured shell. Since the joint is not hollow, an inner mold is not needed. Because the joint has no copper mesh, it is casted using hard polyurethane (Task 9, Smooth-On, Easton, PA) to reduce creep. In comparison, the shell and fingertip were casted using a somewhat softer polyurethane (Task 3, Smooth-On) to enhance impact resistance. Fig. 5 shows the molds and embedded copper mesh prepared for the modified SDM process. After each step, the polyurethane is cured at room temperature for 2-3 days.

\section{STATIC AND DYNAMIC CHARACTERIZATION}

The finger prototype was characterized with respect to static forces, modes of vibration, hysteresis, and thermal effects.

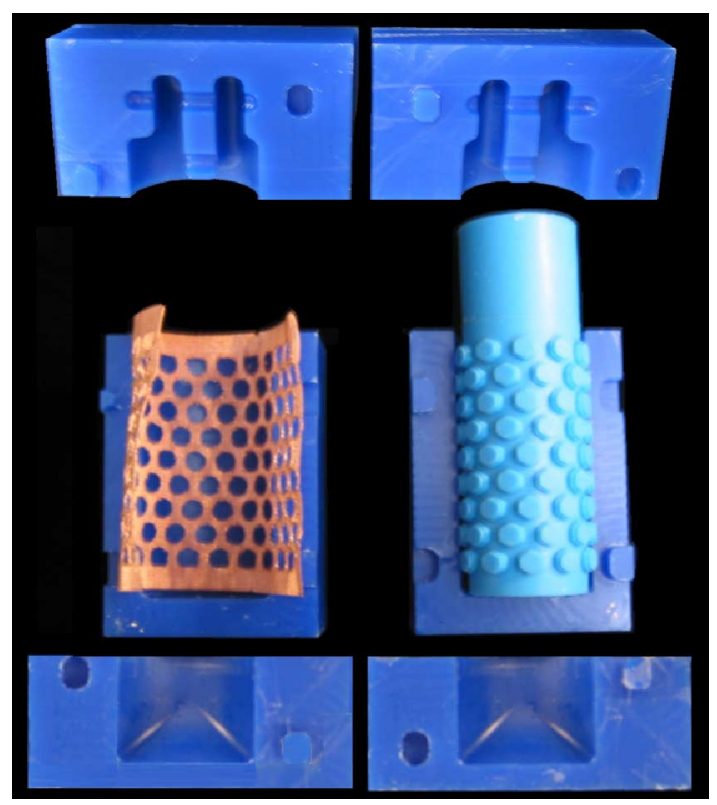

Fig. 5. Wax and silicone rubber molds and copper mesh used in modified SDM fabrication process.
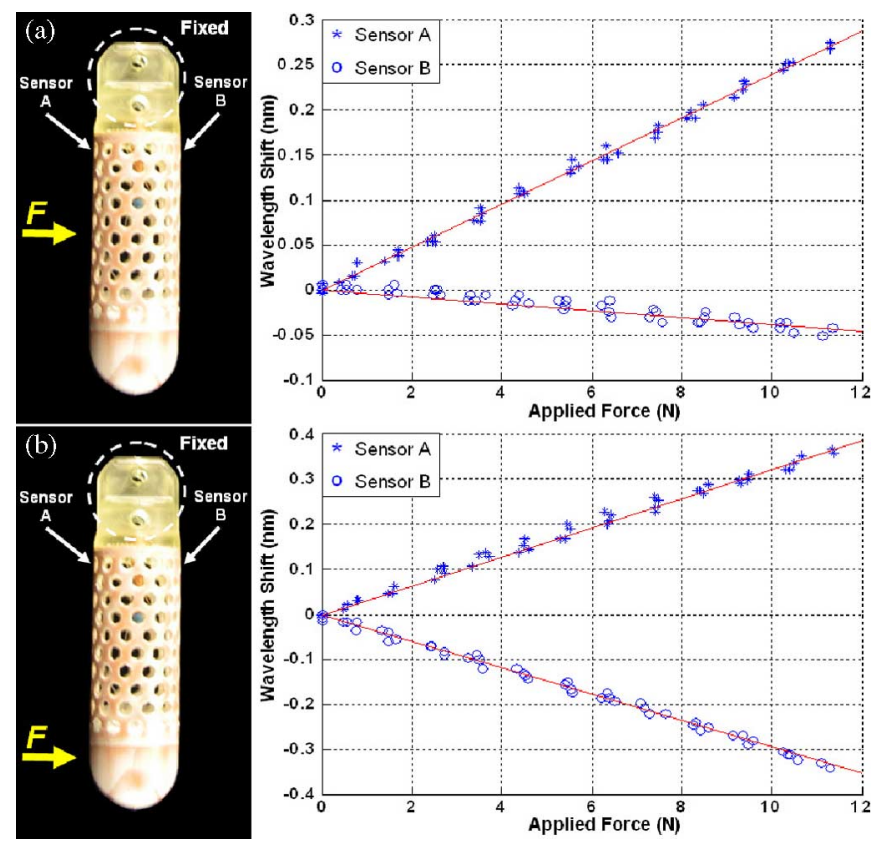

Fig. 6. Static force response results. (a) Shell force response. (b) Fingertip force response.

\section{A. Static Force Sensing}

Static forces were applied to two different locations on the shell and fingertip. Fig. 6 shows the force locations and the responses of two sensors $\mathrm{A}$ and $\mathrm{B}$, in the shell. Applying forces to the shell yielded sensitivities of $24 \mathrm{pm} / \mathrm{N}$ and $-4.4 \mathrm{pm} / \mathrm{N}$ for sensors A and B, respectively. Sensor A, being on the same side of the shell as the contact force, had a much higher strain. Applying a force to the fingertip yielded sensitivities of $32 \mathrm{pm} / \mathrm{N}$ and $-29 \mathrm{pm} / \mathrm{N}$ for sensors $\mathrm{A}$ and $\mathrm{B}$, respectively. In this case, the location of the force resulted in roughly equal strains at both 

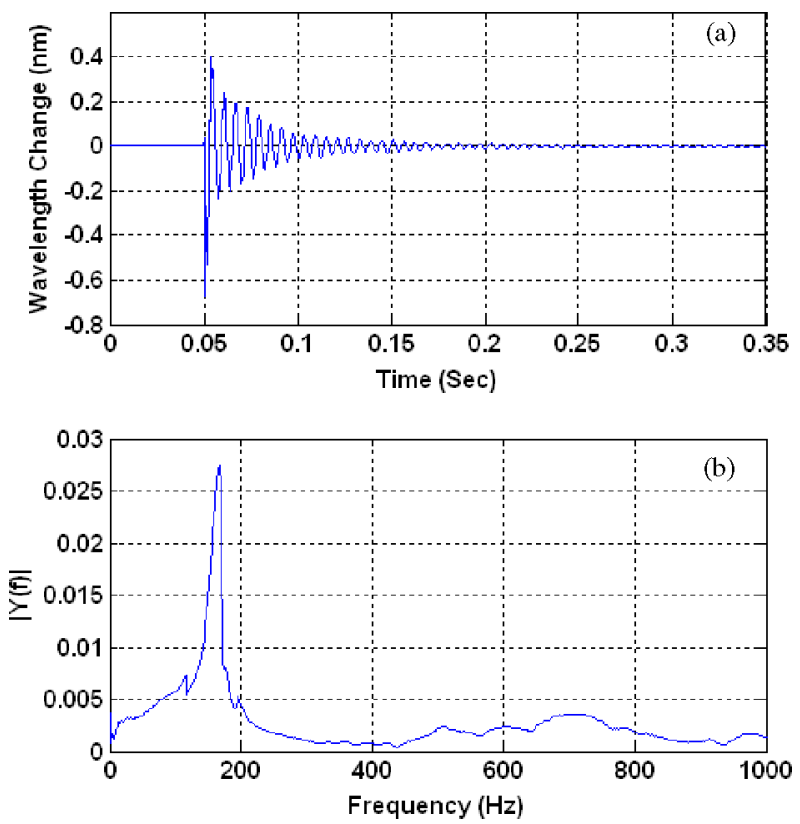

Fig. 7. (a) Impulse response of the finger prototype. (b) Fast Fourier transform of impulse response.

sensors. For a given location, the ratio of the sensor outputs is independent of the magnitude of the applied force. The effect of location is discussed further in Section IV-E. The optical interrogator can resolve wavelength changes of $0.5 \mathrm{pm}$ or less, corresponding to $0.02 \mathrm{~N}$ at the shell and $0.016 \mathrm{~N}$ at the fingertip. However, considering the deviations from linear responses (root means square variations of $5.0 \mathrm{pm}$ and $9.5 \mathrm{pm}$ for the shell and the fingertip tests, respectively), the practical resolutions of force measurement are $0.10 \mathrm{~N}$ at the shell and $0.15 \mathrm{~N}$ at the fingertip. The difference between the minimum detectable force changes and the practical resolution for force sensing are due to a combination of effects including creep in the polymer structure, hysteresis, and thermal drift over the 30-min test cycle. These effects are discussed further in the following sections.

\section{B. Modes of Vibration}

Prior to setting up a closed-loop control system, we investigated the dynamic response of the fingers. Fig. 7 shows the impulse response (expressed as a change in the wavelength of light reflected by an FBG cell) and its fast Fourier transform (FFT). The impulse was effected by tapping on the finger with a light and stiff object: a pencil. The FFT shows a dominant frequency around $167 \mathrm{~Hz}$, which is a result of the dominant vibration mode.

A finite element analysis (see Fig. 8) indicates that there are two dominant vibration modes corresponding to the orthogonal $X$ and $Y$ bending axes, with nearly equal predicted frequencies of just over $180 \mathrm{~Hz}$. The difference between the computed and measured frequency is due to the imperfect modeling of the local stiffness of the polymer/mesh composite. The actual stiffness of the composite depends on manufacturing tolerances, including the location of the mesh fibers within the polymer structure.

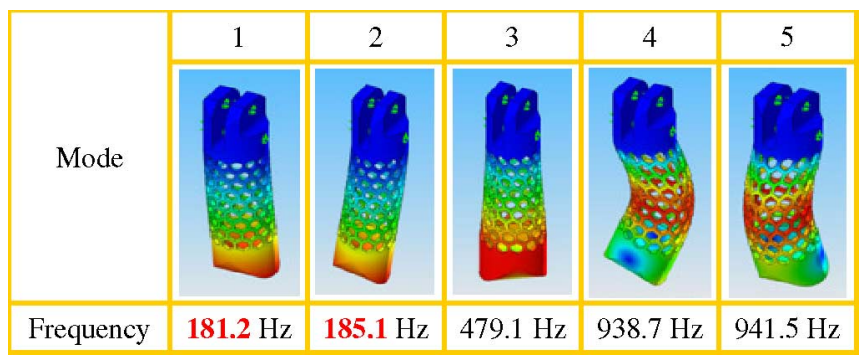

Fig. 8. Modes of vibration of the finger prototype using finite-element analysis. Modes 1 and 2 are the dominant modes, representing bending about $X$ and $Y$ axes, respectively.

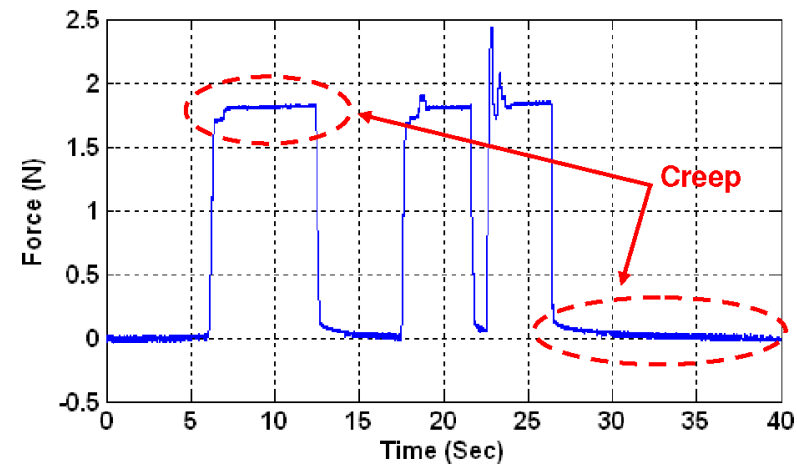

Fig. 9. Effect of applying a steady load for several seconds and then suddenly removing it from the polymer fingertip.
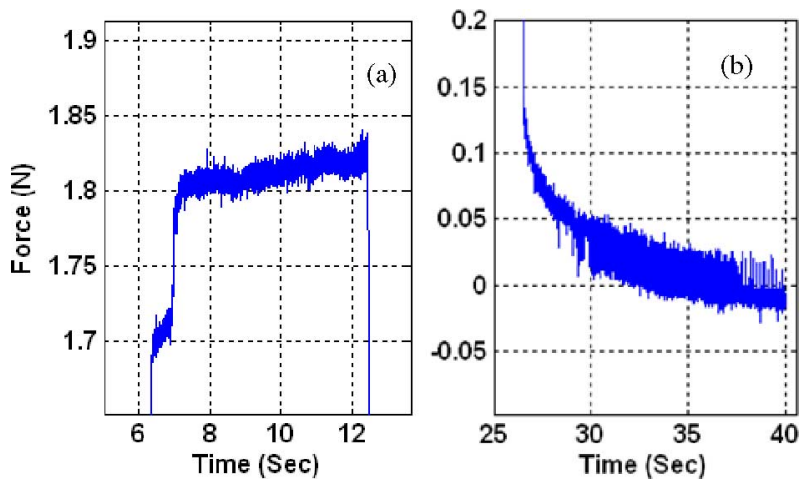

Fig. 10. (a) Detailed views of creep under steady loading and (b) hysteresis associated with sudden unloading.

\section{Hysteresis Analysis}

Polymer structures in general are subject to a certain amount of creep and hysteresis, which is one reason why they have traditionally been avoided for force-sensing and control applications. In the present case, these effects are mitigated by embedding a copper mesh within the structure. However, there is still some creep and hysteresis, as shown in Figs. 9 and 10. The plot in Fig. 9 was produced by applying a moderate load of approximately $1.8 \mathrm{~N}$ to the finger for several seconds and then removing it suddenly. Fig. 10 shows detailed views of loading and unloading periods. The measured force was obtained by optically interrogating the calibrated FBG sensors.

When a steady load is applied for several seconds there is a small amount of creep, part of which also arises from imperfect 


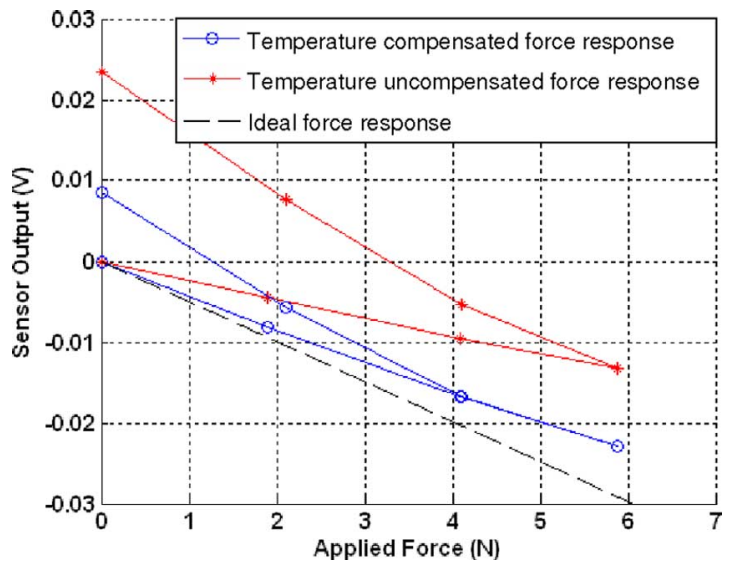

Fig. 11. Test result showing partial temperature compensation provided by the central sensor

thermal compensation. The effect is relatively small over periods of a few seconds, corresponding to typical grasping durations in a pick-and-place or manipulation task. A more significant effect occurs when the load is released. As the plot indicates in Fig. 10(b), the force quickly drops to a value of approximately $0.1 \mathrm{~N}$ and then more slowly approaches zero. To overcome this effect in manipulation tasks, a simple strategy was employed. Whenever the force suddenly dropped to a small value (less than $0.17 \mathrm{~N}$ ), we assumed that contact had been broken. At this point, we reset the zero-offset after a brief time delay. As described in the following section, loss of contact is also a signal to switch the robot from force control to position control.

\section{Temperature Compensation}

Fig. 11 shows a typical thermal test result. Over a 3-min period, the fingertip was loaded and unloaded, while the temperature was decreased from $28.3^{\circ} \mathrm{C}$ to $25.7^{\circ} \mathrm{C}$. The ideal (temperature invariant) sensor output is indicated by the dashed line. The results show that the temperature-compensation sensor reduces the thermal effects. However, a more accurate compensation design is desired in the next prototype.

\section{E. Contact-Force Localization}

It is useful to know the locations of contact forces when a robot is manipulating an object. It is also useful to distinguish, for example, between a desired contact on the fingertip and an unexpected contact elsewhere on the finger. Since the finger prototype has a cylindrical external shape, the location of a contact force can be expressed in terms of latitude and longitude. The following discussion assumes a single contact.

1) Longitudinal Location: Longitudinal localization requires some understanding of the structural deformation of the shell. Fig. 12 shows simplified 2-D diagrams of the prototype. When a force is exerted at a certain location, as shown in (a), the structure will deform, and sensors A and B will measure strains $\varepsilon_{A}$ and $\varepsilon_{B}$, respectively, as indicated. This situation can be decomposed into two separate effects, as shown in (b) and (c). By superposition, $\varepsilon_{A}=\varepsilon_{1}+\varepsilon_{2}$ and $\varepsilon_{B}=\varepsilon_{3}$. Therefore, if the

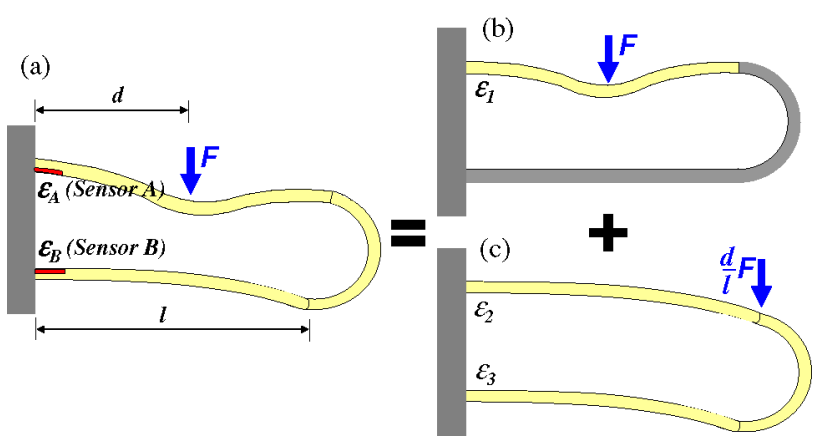

Fig. 12. Two-dimensional simplified shell structure and deformations of finger prototype.

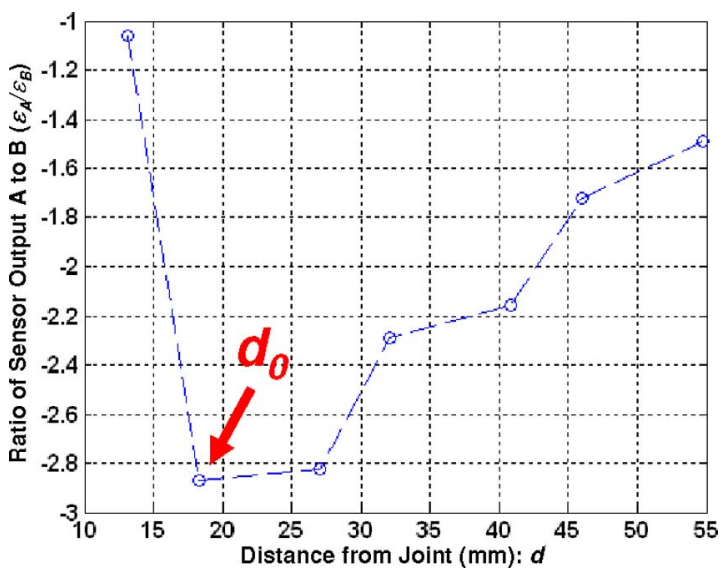

Fig. 13. Strain ratio of sensor A to $B\left(\varepsilon_{A} / \varepsilon_{B}\right)$ for several locations of force application along the length of the finger.
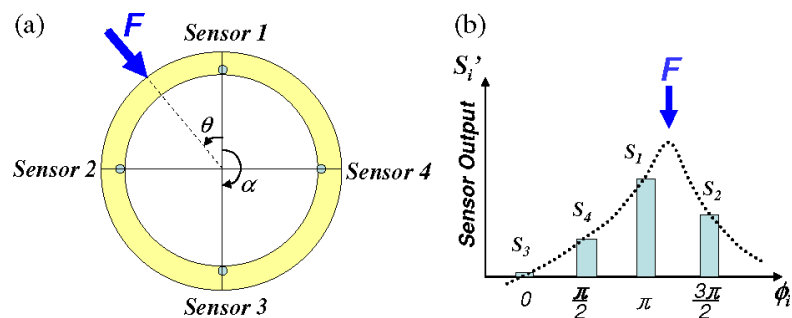

Fig. 14. (a) Top view of the prototype showing embedded sensors and force application. (b) Plot of sensor signal outputs.

ratio of $\varepsilon_{A}$ to $\varepsilon_{B}$ is known, we can estimate $d$, which is the longitudinal force location. Fig. 13 shows the plot of experimental ratios of $\varepsilon_{A}$ to $\varepsilon_{B}$ as a function of $d$.

There is some ambiguity in the localization, since two values of $d$ result in the same ratio. However, if we let $d_{0}$ be the distance at which $\varepsilon_{A} / \varepsilon_{B}$ is minimized and restrict ourselves to the region $d>d_{0}$, then we can resolve this ambiguity. Further, if we modify the manufacturing process to place the sensors closer to the other surface of the shell, then $d_{0}$ approaches 0 , and we can localize an applied force closer to the joint.

2) Latitudinal Location: Latitudinal location can be approximated using centroid and peak detection, as discussed by Son et al. [54]. Fig. 14(a) shows a cross-sectional view of the finger with four strain sensors and an applied contact force indicated. Fig. 14(b) shows its corresponding sensor-signal outputs. The 


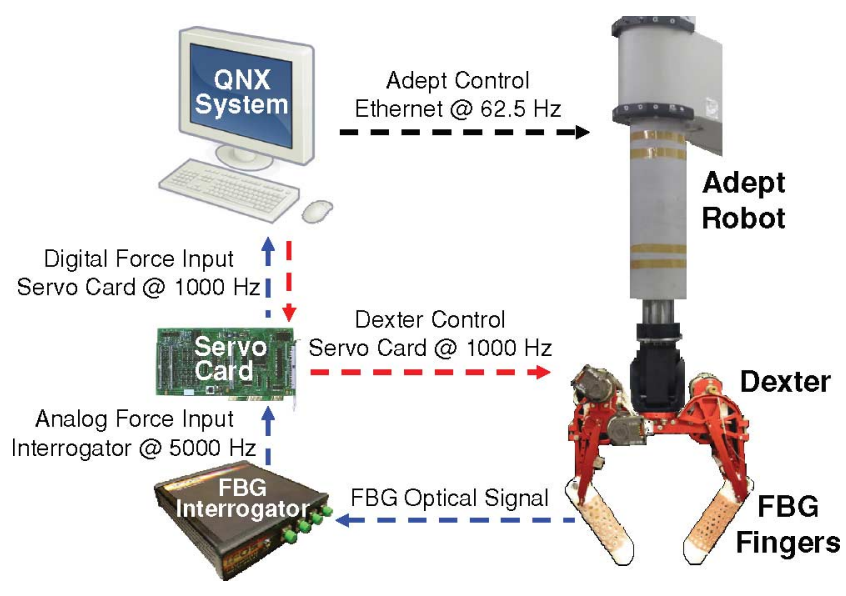

Fig. 15. Hardware system architecture.

two sensors closest to the force location will experience positive strains (positive sensor output), and the other two sensors will experience negative strains (negative sensor output), regardless of the longitudinal location of the force, if $d>d_{0}$. However, since all the sensor signals must be nonnegative to use the centroid method, all signal values must have the minimum signal value subtracted from them. With this, we can find the angular orientation theta of the contact force as

$$
\theta=\frac{\sum \phi_{i} S_{i}^{\prime}}{\sum S_{i}^{\prime}}-\alpha
$$

for $i=1,2,3$, and 4 , where $S_{i}^{\prime}=S_{i}-\min \left\{S_{1}, S_{2}, S_{3}, S_{4}\right\}$, $\phi_{1}=\alpha$, and $\phi_{k}=\phi_{k-1}+\pi / 2$, for $k=2,3,4$ (if $\phi_{k} \geq 2 \pi$, and $\left.\phi_{k}=\phi_{k}-2 \pi\right), S_{i}$ is the output signal from sensor $i$, and $\alpha$ is the clockwise angle between sensor 1 and the sensor with the minimum output-signal value.

This centroid and peak detection method produced errors of less than $2^{\circ}$, which corresponds to less than $0.5 \mathrm{~mm}$ on the perimeter in both finite-element method (FEM) simulation and experiments. However, the experimental data yielded an offset of approximately $5^{\circ}$, while the simulation data yielded an offset of approximately $1.5^{\circ}$. The difference is likely due to manufacturing tolerances in the placement of the sensors.

\section{FORCE CONTROLLER}

Fig. 15 shows the architecture of the hardware system. The two-fingered robot hand, i.e., Dexter, is a low-friction, lowinertia device designed for accurate force control. The hand is controlled by a process running under a real-time operating system (QNX) at $1000 \mathrm{~Hz}$, which reads the joint encoders, computes kinematic and dynamic terms, and produces voltages for linear current amplifiers that drive the motors [20].

The hand controller also acquires force information, via shared memory, from a process that obtains analog force information at $5 \mathrm{kHz}$ from the optical interrogator (I*Sense, IFOS, Inc., Santa Clara, CA) that monitors FBG sensors.

The FBG interrogator is based on high-speed parallel processing using wavelength division multiplexing (WDM). Mul-

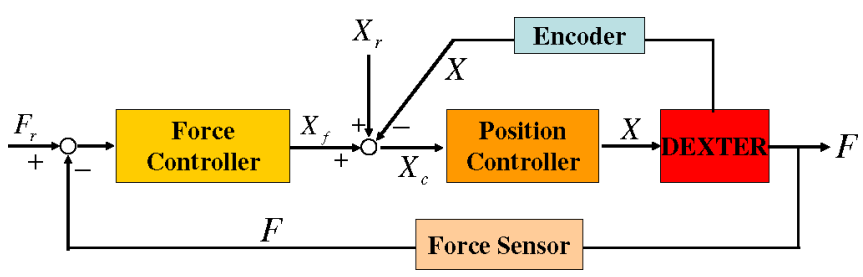

Fig. 16. Position-based force-control system. $F$ and $F_{r}$ are the contact force and user-specified force set point. $X, X_{c}, X_{f}$, and $X_{r}$ are, respectively, the actual position, the commanded position, the position perturbation computed by the force controller, and the reference position of the end-effector.

tiple FBG sensors are addressed by spectral slicing, with the available source spectrum divided up so that each sensor is addressed by a different part of the spectrum. The interrogator built for this study uses 16 channels of a parallel optical-processing chip. Each channel is separated by $100 \mathrm{GHz}$ (approximately $0.8 \mathrm{~nm}$ wavelength spacing around an operating wavelength of $1550 \mathrm{~nm})^{1}$ so that the total required source bandwidth is $12.8 \mathrm{~nm}$. We provide further description of operating principles in Section VIII and describe how this approach can be adapted to support larger numbers of FBG sensors in a single fiber in the Appendix.

Dexter is mounted to a commercial AdeptOne-MV five-axis industrial robot. Communication with the Adept robot is performed using the ALTER software package, which allows new positions to be sent to the Adept robot over an Ethernet connection every $16 \mathrm{~ms}(62.5 \mathrm{~Hz})$. Due to this limitation, all force control is done within Dexter, and the Adept robot is used only for large motions and to keep Dexter approximately centered in the middle of the workspace.

When the fingers are not in contact with an object, the fingers are operated under computed-torque position control, with realtime compensation for gravity torques and inertial terms. When in contact, the fingers are switched over to a nonlinear force control, as described in the next section.

\section{CONTACT FORCE CONTROL}

Most implementations of contact force control can be divided into two categories: impedance control and direct force control [62]. The impedance control [23], [27] aims to control position and force by establishing desired contact dynamics. Force control [46] commands the system to directly track a force set point. For this paper, we adopted a nonlinear controller presented by our collaborator at the National Aeronautics and Space Administration (NASA): the late H. Seraji [49]-[51]. When the system detects contact with the fingertip, it switches to force control, as depicted in Fig. 16. The system actually performs hybrid force/position control [32], [46] at this stage, as the position and force controllers are combined to control forces. The proportional-integral (PI) force controller is constructed as

$$
K(s)=k_{p}+\frac{k_{i}}{s}
$$

\footnotetext{
${ }^{1}$ Operation is in the 1550-nm wavelength window (and, more specifically, within the C-band) to exploit the availability and low cost of components for telecom applications.
} 
based on the first-order admittance

$$
Y(s)=k_{p} s+k_{i}
$$

where $k_{p}$ and $k_{i}$ are the proportional and integral force-feedback gains, respectively. To make the force controller simple, we fix the proportional gain $k_{p}$ to a constant, and make the integral gain $k_{i}$ a nonlinear function of the force error. The nonlinear integral gain is determined by the sigmoidal function

$$
k_{i}=k_{0}+\frac{k_{1}}{1+\exp \left[-\operatorname{sgn}(\Delta) k_{2} e\right]}
$$

where $e$ is the force error $\left(F_{r}-F\right), \Delta=F_{r}-F_{s}, F_{s}$ is the steady value of the contact force before applying new $F_{r}$, and $k_{0}, k_{1}$, and $k_{2}$ are user-specified positive constants that determine the minimum value, the range of variation, and the rate of variation of $k_{i}$, respectively. The value of $\operatorname{sgn}(\Delta)$ is +1 when $F_{r}>F_{s}$, and -1 when $F_{r}<F_{s}$.

We can achieve fast responses and small oscillations in control with this nonlinear gain since the nonlinearity provides high gains with large errors and low gains with small errors. To minimize oscillations due to large proportional gains when the switch occurs between position and force control, all gains, except for the integral force-feedback gain, are ramped from zero to the defined values over a transition time of $0.1 \mathrm{~s}$.

\section{A. Results of Experiments}

In this section, we present the results of two experiments that assess the accuracy of control achieved with the finger prototype. The first experiment shows how accurately the manipulator maintains a desired force during contact by comparing the force data from the prototype with that from a commercial six-axis force-torque sensor (ATI-Nano25 from ATI Industrial Automation). The second experiment shows force control during manipulation tasks, which includes linear and rotational motions of the hand, while grasping an object.

1) Experiment 1 (Force Setpoint Tracking): The Adept arm moves in one direction until the fingertip touches the commercial load cell. As soon as the finger detects contact, the Adept arm stops and the Dexter hand switches to force control. After a period of time, the Adept arm moves away from the object, and the hand switches back to position control. Fig. 17 shows the horizontal motion of the Adept arm in parallel with the joint rotation of the distal joint of the Dexter hand and the force data from both the finger and the load cell. The result shows that the force data from the finger and the load cell almost match exactly over the duration of the experiment. In addition, there is a small amount of slippage reflected in the mirror-image dynamic-force signals reported by the finger and load cell, respectively, as the finger breaks the contact.

We note that to complete the experiment, it was necessary to carefully shield and ground all wires emanating from the commercial load cell due to the large magnetic fields produced by the industrial robot.

2) Experiment 2(Force Control During Manipulation): This experiment concerns the ability of the hand to maintain a desired grasp force while subjected to motions in a manipulation task.

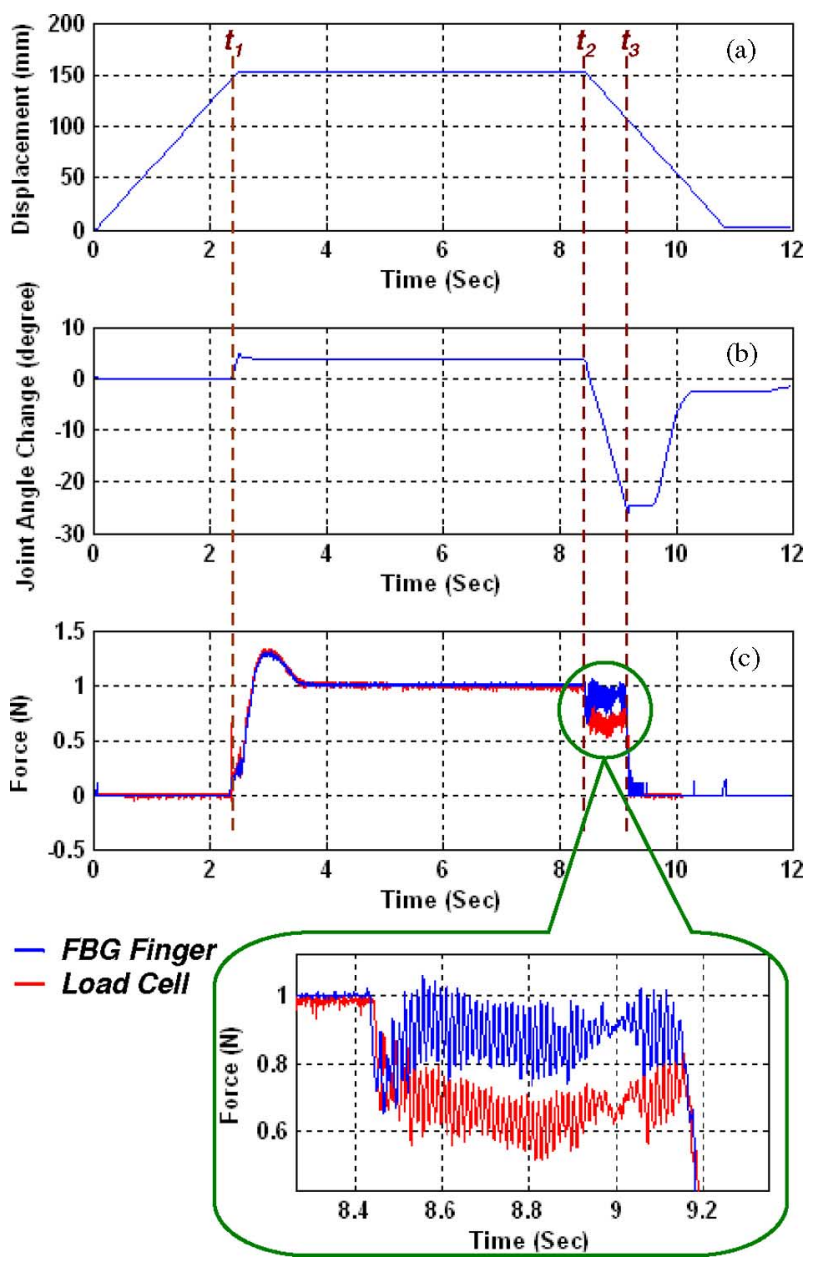

Fig. 17. Experimental results of force set-point tracking. (a) Adept robot motion. (b) Joint angle change of Dexter manipulator. (c) Force data from load cell and FBG-embedded robot-finger prototype. Robot starts force control as soon as it makes a contact with the object at $t_{1}$. Robot starts to retreat at $t_{2}$. Robot breaks contact at $t_{3}$.

The robot was commanded to lift the grasped object, which is a metal block weighing $100 \mathrm{~g}$, move it horizontally to a distance of approximately $30 \mathrm{~cm}$, rotate it about the $Z$ and $Y$ axes, return the block to the original location, and replace it. In every case, the controller returned to the desired force within $0.01 \mathrm{~s}$. The results of this experiment can be seen in Fig. 18. The magnitude of the combined ( $X, Y$, and $Z$ ) acceleration of the manipulator is plotted in parallel with the measured grasp force. Disturbances associated with the accelerations and decelerations along the path can be observed in the force data. The rms of force errors during the force control is $<0.03 \mathrm{~N}$.

Since the current finger prototype is capable of control oneaxis forces, more complicated force control experiments, in two or three axes, will be carried out in the future.

\section{MinIATURIZED ForCE-SENSING FINGER}

Following the successful creation of large-scale $(120 \mathrm{~mm}$ long) robot fingers, the next step was to produce human-scale fingertips for robots designed for human interaction in space. 

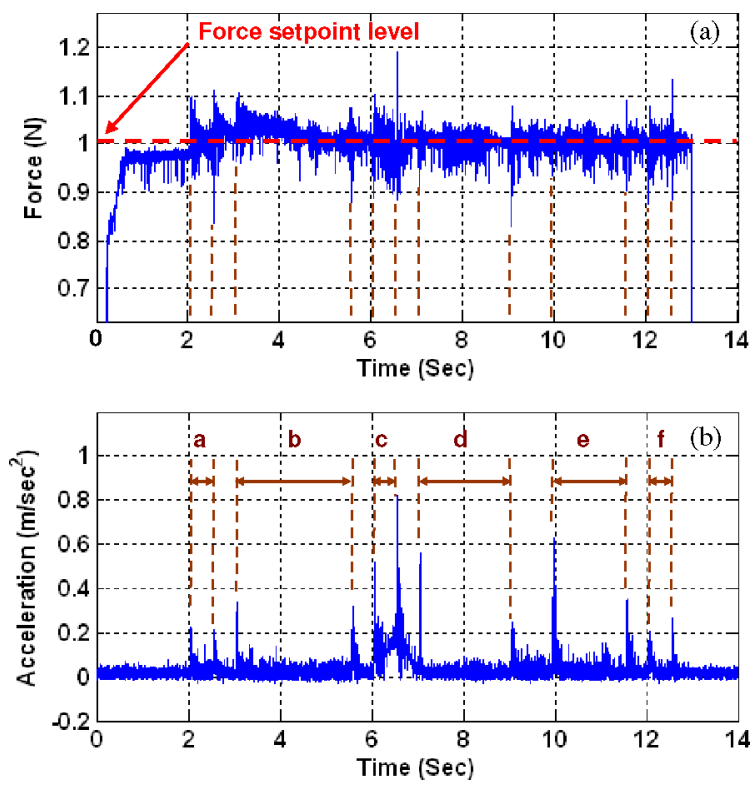

Fig. 18. Experimental results of force control during manipulation tasks. (a) Grasp force measured by a finger with FBG sensors. (b) Acceleration plotted along with magnitude of combined $(X, Y$, and $Z)$ acceleration of the robot. Periods $\mathrm{a}, \mathrm{b}, \mathrm{e}$, and $\mathrm{f}$ are for translation motions. Periods $\mathrm{c}$ and $\mathrm{d}$ are for rotation motions. Every task motion is followed by a waiting period before starting the next motions.

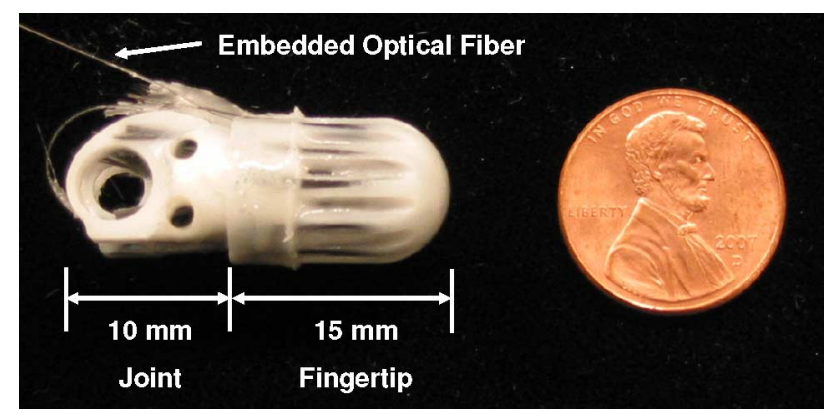

Fig. 19. Miniaturized polyurethane finger prototype fabricated as a hollow shell composed of several curved ribs that are connected at the base by a circular ring that meet at the apex. One optical fiber with four FBG sensors is embedded in the ribs. The structure is reinforced with embedded carbon fibers.

The same technology, which has no metal components or electronics, could also be applied to robots for MRI procedures.

Fig. 19 shows a prototype of a small fingertip with an embedded optical fiber containing FBG strain sensors. For this application, an $80-\mu \mathrm{m}$-diameter bend-insensitive optical fiber from OFS was selected. These fibers tolerate comparatively tightbending radii (approximately $7.5 \mathrm{~mm}$ ). In addition to the optical fibers, carbon fiber was embedded for structural reinforcement and creep reduction.

Fig. 20 shows the results of force-calibration tests. Applying force up to approximately $5 \mathrm{~N}$ to the fingertip yielded sensitivities of 71, 54, and $7.2 \mathrm{pm} / \mathrm{N}$ in $X, Y$, and $Z$ axes, respectively. Considering the wavelength resolution of the optical interrogator, which is better than $0.5 \mathrm{pm}$, the minimum detectable force changes are less than $0.01 \mathrm{~N}$ in $X$ and $Y$ axes and $0.07 \mathrm{~N}$ in the $Z$ axis, assuming no temperature changes. The practical resolutions of force measurement are $0.05 \mathrm{~N}$ in the $X$ and $Y$ axes
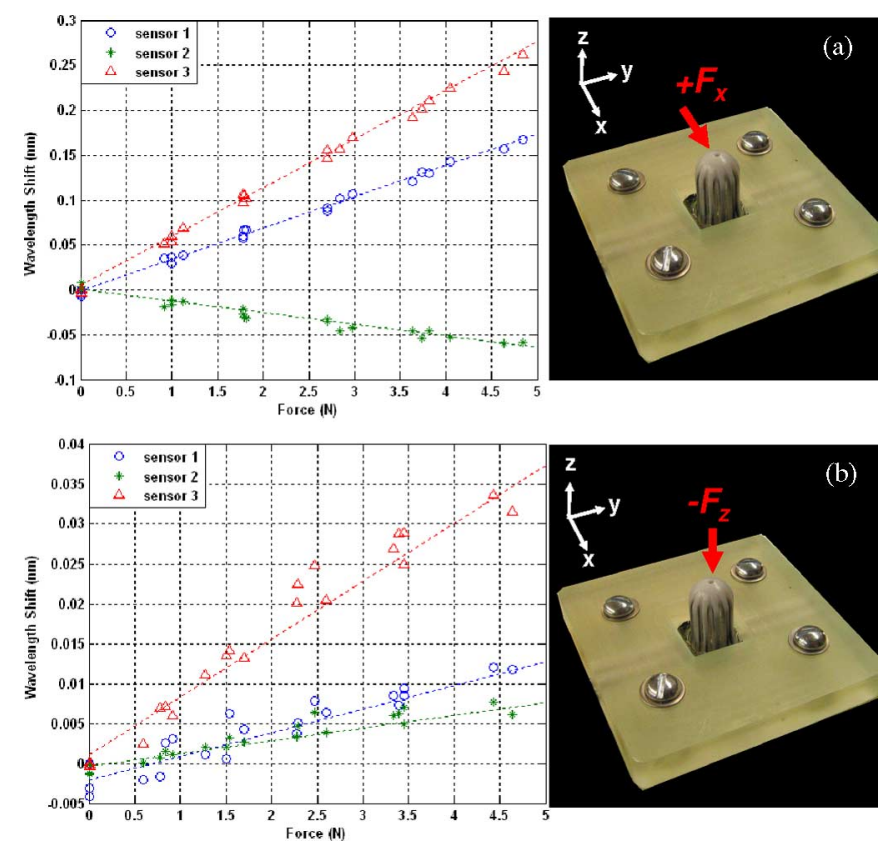

Fig. 20. Calibration results. (a) $X$-axis force response ( $Y$ is similar). (b) $Z$-axis force response.

and $0.16 \mathrm{~N}$ in the $Z$ axis, considering deviations from linearity. Although the current prototype does not contain a temperature compensation sensor, future designs will address temperature compensation, as well as increased axial ( $Z$-axis) sensitivity.

\section{OPTICAL-INTERROGATION SYSTEM}

The overall interrogator architecture follows the one presented in [39], except that the photonic processor in the present case is based on an arrayed waveguide grating (AWG) technology [40], [48], [59], which has been customized for this application [6]. The approach is based on a parallel photonicprocessing architecture that has the near-term potential to combine high channel counts ( $>100$ sensors on a single fiber), high resolution (sub- $\mu \varepsilon$ ), and high speed $(>5 \mathrm{kHz}$ ) with a miniaturized footprint. These features will become valuable as we seek to augment the sensor number and response speed of our robot system. The ultimate goal is to have the interrogator integrated into the robotic structure.

As previously discussed, the application of strain on each FBG produces a shift in the selected wavelength, which the interrogator measures. Interrogators can be tunable (examining each FBG sequentially) or parallel in nature. The latter approach, which forms the basis of the our system, has advantages in terms of speed, particularly with many sensors.

The interrogator combines (a) optical signal processing (broadband light source, optical circulator, passive photonic parallel processing chip, and photodetector array) with (b) postdetection electronics, and (c) control and monitoring subsystems, as shown in Fig. 21. Operation is as follows.

1) The broadband source sends light through the optical circulator to an array of FBGs, each of which reflects a different Bragg wavelength. 


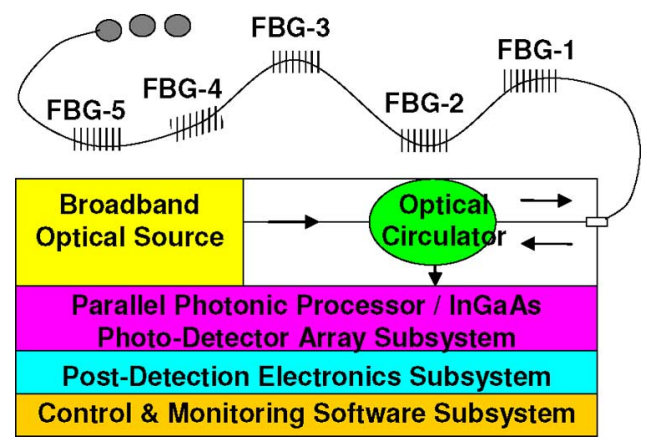

Fig. 21. Functional diagram of FBG interrogator based on a photonic parallel spectral processor that simultaneously processes signals reflected from all FBGs.

2) The reflected light is returned through the optical circulator to the photonic processor.

3) The parallel photonic processor demultiplexes the light into multiple wavelength channels and provides the basis for a ratiometric approach to measure each of the returned strain-dependent wavelengths.

4) The returned wavelengths are converted to arrayed electrical signals by the multichannel photodetector array.

5) Electronics and software provide the final conversion of the arrayed signals to wavelengths and the strains.

The parallel photonic processor used in our interrogator is based on planar lightwave circuit (PLC) and phased-array technology. Optical (and potentially optoelectronic) integration technology allows for fabrication of the photonic processor as a single mass-producible multifunctional chip. This approach is central to achieve the cost and size reductions that will bring FBG sensing solutions into widespread usage.

\section{CONCLUSION AND FUTURE WORK}

This paper has described the development of exoskeletal force-sensing robot fingers using embedded FBG optical sensors. A rapid prototyping process, which is called the shape deposition manufacturing, was modified to support the fabrication of hollow, plastic mesh structures with embedded components. The sensors were embedded near the base for high sensitivity to imposed loads. The resulting structure is light and rugged. In initial experiments, the sensorized structure demonstrated minimum detectable force changes of less than $0.02 \mathrm{~N}$ and practical force measurement resolutions of less than $0.15 \mathrm{~N}$, as well as a dominant frequency at $167 \mathrm{~Hz}$. With more precise location of the sensors, higher sensitivities should be possible in the future. We also note that any frequency limit is provided by the mechanical finger system and not by the interrogator that can measure dynamic strains to $5 \mathrm{kHz}$.

A copper mesh in the structure reduces viscoelastic creep and provides thermal shielding. A single FBG temperature compensation sensor at the center of the hollow finger helps to reduce the overall sensitivity to thermal variations. However, the central sensor is sufficiently distant from the exterior sensors so that changes in temperature produce noticeable transient signals. This effect can be reduced in the future by using a larger number of sensors and locating thermal compensation sensors near the exterior of the structure, where they undergo the same transient thermal strains as the other sensors.

Experiments were also conducted to investigate the finger prototype's ability to localize contact forces. Although the ability to localize forces with just four exterior sensors is limited, the results show that the mesh does respond globally to point contacts in a predictable way. With a larger number of sensors, more accurate contact localization will be possible. Increasing the number of sensors is relatively straightforward, as multiple FBGs can be located along each fiber with multiplexing.

A robot hand with the finger prototypes was operated in a hybrid control scheme. The finger sensors are capable of resolving small forces, and are immune to electromagnetic disturbances, so that the system can be mounted on a large industrial robot or in other applications where large magnetic fields are present, without concern for shielding and grounding. In addition, as multiple FBG sensors can be placed along a single fiber and multiplexed optically, it suffices to route a single fiber down the robot arm. The potential to miniaturize the technology is demonstrated with a second prototype having dimensions comparable to a human fingertip. Future versions of this prototype will incorporate additional sensors for thermal compensation and a modified design for greater sensitivity to axial loads.

In parallel, we have been developing versions of the interrogator [33], [39] to support larger numbers of sensors with high resolution and long-term stability. Some design considerations are discussed in the Appendix. As the FBG technology evolves, we foresee the potential in robotics for bend sensors based on multicore fibers, as well as the use of polymer FBGs [14] in flexible robotic skins. Another possibility is to use multiparameter dual Bragg gratings in a polarization-maintaining fiber for multiaxial strain measurements [36].

\section{APPENDIX}

For the range of broadband light sources that we use, the available source bandwidth is between 40 and $100 \mathrm{~nm}$. Thus, if we make use of the entire available source spectrum and allocate $2 \mathrm{~nm}$ per sensor, then we can support 20-50 sensors on a single fiber. This number can be increased by using multiple fibers. More precisely, the number of sensors $N_{\text {sensors }}$ that can be supported on a single fiber is related to the source bandwidth, $\delta \lambda_{\text {source }}$ divided by the bandwidth required for each sensor $\delta \lambda_{\text {sensor }}$. Further, $\delta \lambda_{\text {sensor }}$ is given by the maximum straindependent wavelength shift $\delta \lambda_{\text {strain-max }}$, and the sensor wavelength separation, to avoid crosstalk (i.e., to keep it below a "tolerable" level, i.e., $\delta \lambda_{\text {crosstalk }}$ ). Thus

$$
N_{\text {sensors }}=\frac{\delta \lambda_{\text {source }}-\left|\delta \lambda_{T U}\right|}{\delta \lambda_{\text {strain-max }}+\delta \lambda_{\text {crosstalk }}+\delta \lambda_{T N}} .
$$

If the photonic processor is maintained at a constant temperature, while the FBGs see a varying temperature, then the effective source bandwidth if reduced by the term $\delta \lambda_{T U}$, which is the maximum FBG wavelength shift due to temperature change, is typically $10 \mathrm{pm} /{ }^{\circ} \mathrm{C}$. Thus, for a $100^{\circ} \mathrm{C}$ temperature change, this term results in a $10 \%$ reduction in $N_{\text {sensors }}$ for $\delta \lambda_{\text {source }}=$ $100 \mathrm{~nm}$. If all sensors see the same temperature variation, then 
TABLE II

TYPICAL SENSOR NUMBERS THAT CAN BE SUPPORTED FOR A RANGE OF SPECTRAL CHARACTERISTICS AND STRAIN REQUIREMENTS

\begin{tabular}{ccccc}
\hline \hline$\delta \lambda_{\text {source }}$ & $\delta \lambda_{\text {strain-max }}$ & $N_{\text {sensors }}$ & & \\
\hline & & $\delta \lambda_{T U}=0^{\circ} \mathrm{C}$ & $100^{\circ} \mathrm{C}$ & $100^{\circ} \mathrm{C}$ \\
& & $\delta \lambda_{T N}=0^{\circ} \mathrm{C}$ & $0{ }^{\circ} \mathrm{C}$ & $10^{\circ} \mathrm{C}$ \\
\hline \multirow{2}{*}{$100 \mathrm{~nm}$} & $1.2 \mathrm{~nm}(\rightarrow 1000 \mu \varepsilon)$ & 50 & 45 & 30 \\
& $9.2 \mathrm{~nm}(\rightarrow 7700 \mu \varepsilon)$ & 10 & 9 & 8 \\
\hline \multirow{2}{*}{$40 \mathrm{~nm}$} & $1.2 \mathrm{~nm}(\rightarrow 1000 \mu \varepsilon)$ & 20 & 15 & 10 \\
& $9.2 \mathrm{~nm}(\rightarrow 7700 \mu \varepsilon)$ & 4 & 3 & 2 \\
\hline \hline
\end{tabular}

they shift uniformly with temperature. On the other hand, if sensors that are adjacent in wavelength see different temperatures, then the spacing needs to be increased by $\delta \lambda_{T N}$, the nonuniform, or differential temperature-dependent wavelength shift. For $10{ }^{\circ} \mathrm{C}$ variation between sensors, $N_{\text {sensors }}$ decreases by one third.

The wavelength separation to avoid crosstalk $\delta \lambda_{\text {crosstalk }}$ (to the extent that wavelength change in one grating does not produce a "measurable" change in the wavelength computed for the adjacent grating) will depend on the FBG spectrum and the parallel-spectral-processor channel spectra (spacing, bandwidths, and shape), as well as the desired measurement precision but is typically on the order of one to two times the channel separation. Table II summarizes the possible sensor numbers for different source bandwidths and maximum strain-dependent wavelength shifts assuming $0.8 \mathrm{~nm}$ for the parallel processor wavelength separation and $\delta \lambda_{\text {crosstalk }}$.

\section{ACKNOWLEDGMENT}

The authors would like to thank NASA technical monitor T. Martin, for his support and feedback, and late Dr. H. Seraji of NASA's Jet Propulsion Laboratory for his contributions to the project.

\section{REFERENCES}

[1] M. Amano, Y. Okabe, N. Takeda, and T. Ozaki, "Structural health monitoring of an advanced grid structure with embedded fiber Bragg grating sensors," Struct. Health Monit., vol. 6, no. 4, pp. 309-324, 2007.

[2] L. Ascari, P. Corradi, L. Beccai, and C. Laschi, "A miniaturized and flexible optoelectronic sensing system for a tactile skin," Int. J. Micromech. Microeng., vol. 17, pp. 2288-2298, 2007.

[3] F. G. Barth, "Spider mechanoreceptors," Curr. Opin. Neurobiol., vol. 14, pp. 415-422, 2004.

[4] F. G. Barth and J. Stagl, "The slit sense organs of arachnids," Zoomorphologie, vol. 86, pp. 1-23, 1976.

[5] A. Bicchi and G. Canepa, "Optimal design of multivariate sensors," Meas. Sci. Technol., vol. 5, pp. 319-332, 1994.

[6] R. J. Black and B. Moslehi, "Fiber Bragg grating interrogators for structural health monitoring," presented at the Soc. Adv. Mater. Process. Eng., Long Beach, CA, 2008.

[7] R. J. Black, D. Zare, L. Oblea, Y.-L. Park, B. Moslehi, and C. Neslen, "On the gage factor for optical fiber grating strain gages," presented at the Soc. Adv. Mater. Process Eng., Long Beach, CA, 2008.

[8] R. Blickhan and F. G. Barth, "Strains in the exoskeleton of spiders," $J$. Comp. Physiol. A, vol. 157, pp. 115-147, 1985.

[9] W. Bluethmann, R. Ambrose, M. Diftler, S. Askew, E. Huber, M. Goza, F. Rehnmark, C. Lovchik, and D. Magruder, "Robonaut: A Robot designed to work with humans in space," Auton. Robots, vol. 14, pp. 179-197, 2003.

[10] L. Carvalho, J. C. C. Silva, R. N. Nogueira, J. L. Pinto, H. J. Kalinowski, and J. A. Simöes, "Application of Bragg grating sensors in dental biomechanics," J. Strain Anal. Eng. Des., vol. 41, no. 6, pp. 411-416, 2006.
[11] L. A. Danisch, K. Englehart, and A. Trivett, "Spatially continuous six degree of freedom position and orientation sensor," Sens. Rev., vol. 19, no. 2, pp. 106-112, 1999.

[12] L. A. Danisch and E. M. Reimer, "Pressure sensor based on illumination of a deformable integrating cavity," U.S. Patent, 5917 180, 1999.

[13] C. R. Dennison, P. M. Wild, M. F. Dvorak, D. R. Wilson, and P. A. Cripton, "Validation of a novel minimally invasive intervertebral disc pressure sensor utilizing in-fiber Bragg gratings in a porcine model: An ex vivo study," Spine, vol. 33, no. 17, pp. E589-E594, 2008.

[14] H. Dobb, D. J. Webb, K. Kalli, A. Argyros, M. C. J. Large, and M. A. van Eijkelenborg, "Continuous wave ultraviolet light-induced fibre Bragg gratings in few-and single-moded microstructured polymer optical fibres," Opt. Lett., vol. 30, no. 24, pp. 3296-3298, 2006.

[15] A. Dollar, C. R. Wagner, and R. D. Howe, "Embedded sensors for biomimetic robotics via shape deposition manufacturing," in Proc. 1st IEEE/RAS-EMBS Int. Conf. Biomed. Robot. Biomechatron., 2006 pp. 763-768

[16] W. Ecke, I. Latka, R. Willsch, A. Reutlinger, and R. Graue, "Fiber optic sensor network for spacecraft health monitoring," Meas. Sci. Technol., vol. 12, no. 7, pp. 974-980, 2001.

[17] A. F. Fernandez, F. Berghmans, B. Brichard, P. Mégret, M. Decréton, M. Blondel, and A. Delchambre, "Multi-component force sensor based on multiplexed fibre Bragg grating strain sensors," Meas. Sci. Technol., vol. 12, pp. 810-813, 2001.

[18] R. F. Foelix, Biology of Spiders, 2nd ed. New York: Oxford Univ. Press, 1996.

[19] E. J. Friebele, C. G. Askins, A. B. Bosse, A. D. Kersey, H. J. Patrick, W. R. Pogue, M. A. Putnam, W. R. Simon, F. A. Tasker, W. S. Vincent, and S. T. Vohra, "Optical fiber sensors for spacecraft applications," Smart Mater. Struct., vol. 8, pp. 813-838, 1999.

[20] W. Griffin, W. M. Provancher, and M. R. Cutkosky, "Feedback strategies for telemanipulation with shared control of object handling forces," Presence: Teleoper. Vir. Environ., vol. 14, no. 6, pp. 720-731, 2005.

[21] T. C. Hales. (1999). The honeycomb conjecture [Online]. Available: http://arxiv.org/abs/math.MG/9906042

[22] K. O. Hill and G. Meltz, "Fiber Bragg grating technology fundamentals and overview," J. Lightw. Technol., vol. 15, no. 8, pp. 1263-1276, Aug. 1997.

[23] N. Hogan, "Impedence control: An approach to manipulation, Parts I-III," ASME J. Dyn. Syst., Meas., Control, vol. 107, no. 1, pp. 1-24, 1985.

[24] J. Hong and X. Tan, "Calibrating a VPL data glove for teleoperating the Utah/MIT hand," in Proc. IEEE Int. Conf. Robot. Autom., 1989, vol. 3, pp. 1752-1757.

[25] J. Jung, H. Nam, B. Lee, J. O. Byun, and N. S. Kim, "Fiber Bragg grating temperature sensor with controllable sensitivity," Appl. Opt., vol. 38, no. 13 , pp. 2752-2754, 1999.

[26] K. Kamiyama, H. Kajimoto, M. Inami, N. Kawakami, and S. Tachi, "Development of a vision-based tactile sensor," IEEJ Trans. Sens. Micromach., vol. 123, no. 1, pp. 16-22, 2003.

[27] H. Kazernooni, T. B. Sheridan, and P. K. Houpt, "Robust compliant motion for manipulators, Parts I-II," IEEE Trans. Robot. Autom., vol. RA-2, no. 2, pp. 83-105, Jun. 1986.

[28] A. D. Kersey, M. A. Davis, H. J. Patrick, M. LeBlanc, K. P. Koo, C. G. Askins, M. A. Putnam, and E. J. Friebele, "Fiber grating sensors," $J$. Lightw. Technol., vol. 15, no. 8, pp. 1442-1463, Aug. 1997.

[29] J. M. Ko and Y. Q. Ni, "Technology developments in structural health monitoring of large-scale bridges," Eng. Struct., vol. 27, no. 12, pp. 17151725, 2005.

[30] H.-N. Li, D.-S. Li, and G.-B. Song, "Recent applications of fiber optic sensors to health monitoring in civil engineering," Eng. Struct., vol. 26, no. 11, pp. 1647-1657, 2004.

[31] X. C. Li and F. Prinz, "Metal embedded fiber Bragg grating sensors in layered manufacturing," J. Manuf. Sci. Eng., vol. 125, pp. 577-585, 2003.

[32] G. Liu and Z. Li, "A unified geometric approach to modeling and control of constrained mechanical systems," IEEE Trans. Robot. Autom., vol. 18, no. 4, pp. 574-587, Aug. 2002.

[33] C. Lopatin, E. Mendez, B. Moslehi, R. J. Black, K. Chau, and L. Oblea, "Progress in miniaturization of a multichannel optical fiber Bragg grating sensor interrogator," in Proc. SPIE 3rd Eur. Workshop Opt. Fibre Sens., 2007, vol. 6619, pp. 66193x1-66193x4.

[34] H. Maekawa, K. Tanie, and K. Komoriya, "Tactile feedback for multifingered dynamic grasping," IEEE Control Syst. Mag., vol. 17, no. 1, pp. 63-71, Feb. 1997.

[35] S. Magne, S. Rougeault, M. Vilela, and P. Ferdinand, "State-of-strain evaluation with fiber Bragg grating rosettes: Application to discrimination 
between strain and temperature effects in fiber sensors," Appl. Opt. vol. 36, no. 36, pp. 9437-9447, 1997.

[36] T. Mawatari and D. Nelson, "A multi-parameter Bragg grating fiber optic sensor and triaxial strain measurement," Smart Mater. Struct., vol. 17, pp. $1-19,2008$.

[37] D. T. Moran, K. M. Chapman, and R. S. Ellis, "The fine structure of cockroach campaniform sensilla," J. Cell Biol., vol. 48, pp. 155-173, 1971.

[38] W. W. Morey, G. Meltz, and J. M. Weiss, "Recent advances in fiber-grating sensors for utility industry applications," in Proc. SPIE, Self-Calibrated Intell. Opt. Sens. Syst., 1996, vol. 2594, pp. 90-98.

[39] B. Moslehi, R. J. Black, K. Toyama, and H. J. Shaw, "Multiplexible fiberoptic strain sensor system with temperature compensation capability," Divisions 1-3, U.S. Patents 6895132 (May 17, 2005), 6788835 (Sep. 7, 2004), 6597822 (Jul. 22, 2003).

[40] D. C. C. Norman, D. J. Webb, and R. D. Pechstedt, "Extended range interrogation of wavelength division multiplexed fiber Bragg grating sensors using arrayed waveguide grating," Electron. Lett., vol. 39, pp. 1714-1715, 2003.

[41] S. Pal, J. Mandal, T. Sun, K. T. V. Grattan, M. Fokine, F. Carlsson, P. Y. Fonjallaz, S. A. Wade, and S. F. Collins, "Characteristics of potential fibre Bragg grating sensor-based devices at elevated temperatures," Meas. Sci. Technol., vol. 14, pp. 1131-1136, 2003.

[42] Y.-L. Park, K. Chau, R. J. Black, and M. R. Cutkosky, "Force sensing robot fingers using embedded fiber Bragg grating sensors and shape deposition manufacturing," in Proc. IEEE Int. Conf. Robot. Autom., 2007, pp. 15101516.

[43] Y.-L. Park, S. Elayaperumal, B. L. Daniel, E. Kaye, K. B. Pauly, R. J. Black, and M. R. Cutkosky, "MRI-compatible haptics: Feasibility of using optical fiber Bragg grating strain-sensors to detect deflection of needles in an MRI environment," presented at the Int. Soc. Magn. Reson. Med., 16th Sci. Meet. Exhib., Toronto, ON, Canada, 2008.

[44] Y.-L. Park, S. C. Ryu, R. J. Black, B. Moslehi, and M. R. Cutkosky, "Fingertip force control with embedded fiber Bragg grating sensors," in Proc. IEEE Int. Conf. Robot. Autom., 2008, pp. 3431-3436.

[45] I. Peterson, "The honeycomb conjecture," Sci. News, vol. 156, no. 4, pp. 60-61, 1999

[46] M. Raibert and J. Craig, "Hybrid position/force control of manipulators," ASME J. Dyn. Syst., Meas., Control, vol. 102, no. 2, pp. 126-133, 1981.

[47] Y. J. Rao, "In-fibre Bragg grating sensors," Meas. Sci. Technol., vol. 8, pp. 355-375, 1997.

[48] Y. Sano and T. Yoshino, "Fast optical wavelength interrogator employing arrayed waveguide grating for distributed fiber Bragg grating sensors," $J$. Lightw. Technol., vol. 21, no. 1, pp. 132-139, Jan. 2003.

[49] H. Seraji, "A new class of nonlinear PID controllers with robotic applications," J. Robot. Syst., vol. 15, no. 3, pp. 161-181, 1998

[50] H. Seraji, D. Lim, and R. Steele, "Experiments in contact control," $J$. Robot. Syst., vol. 13, no. 2, pp. 53-73, 1996.

[51] H. Seraji and R. Steele, "Nonlinear contact control for space station dexterous arms," in Proc. IEEE Int. Conf. Robot. Autom., 1998, vol. 1, pp. 899906.

[52] E.-A. Seyfarth, W. Eckweiler, and K. Hammer, "Proprioceptors and sensory nerves in the legs of a spider, Cupiennius salei (Arachnida, Araneida)," Zoomorphologie, vol. 105, pp. 190-196, 1985.

[53] D. S. Smith, "The fine structure of haltere sensilla in the blowfly Calliphora erythrocephala (Meig.) with scanning electron microscopic observations on the haltere surface," Tissue Cell, vol. 1, pp. 443-484, 1969.

[54] J. S. Son, M. R. Cutkosky, and R. D. Howe, "Comparison of contact sensor localization abilities during manipulation," in Proc. IEEE/RSJ Int. Conf. Intell. Robots Syst., 1995, vol. 2, pp. 96-103.

[55] R. Suresh, S. C. Tjin, and S. Bhalla, "Multi-component force measurement using embedded fiber Bragg grating," Opt. Laser Technol., vol. 41, no. 4, pp. 431-440, 2008. DOI:10.1016/j.optlastec.2008.08.004.

[56] N. Takahashi, A. Hirose, and S. Takahashi, "Underwater acoustic sensor with fiber Bragg grating," Opt. Rev., vol. 4, no. 6, pp. 691-694, 1997.

[57] R. M. Voyles, G. Fedder, and P. K. Khosla, "Design of a modular tactile sensor and actuatator based on an electrorheological gel," in Proc. IEEE Int. Conf. Robot. Autom., 1996, vol. 1, pp. 13-17.

[58] L. E. Weiss, R. Merz, F. B. Prinz, G. Neplotnik, P. Padmanabhan, L. Schultz, and K. Ramaswami, "Shape deposition manufacturing of heterogenous structures," J. Manuf. Syst., vol. 16, no. 4, pp. 239-248, 1997.

[59] G. Z. Xiao, P. Zhao, F. G. Sun, Z. G. Lu, Z. Zhang, and C. P. Grover, "Interrogating fiber Bragg grating sensors by thermally scanning an arrayed waveguide grating based demultiplexer," Opt. Lett., vol. 29, pp. 22222224, 2004.
[60] M. G. Xu, J.-L. Archambault, L. Reekie, and J. P. Dakin, "Discrimination between strain and temperature effects using dual-wavelength fibre grating sensors," Electron. Lett., vol. 30, no. 13, pp. 1085-1087, 1994.

[61] M. G. Xu, L. Dong, L. Reekie, J. A. Tucknott, and J. L. Cruz, "Temperature-independent strain sensor using a chirped Bragg grating in a tapered optical fibre," Electron. Lett., vol. 31, no. 10, pp. 823-825, 1995.

[62] G. Zeng and A. Hemami, "An overview of robot force control," Robotica, vol. 15 , pp. 473-482, 1997.

[63] L. Zhang, J. Qian, Y. Zhang, and L. Shen, "On SDM/WDM FBG sensor net for shape detection of endoscope," in Proc. IEEE Int. Conf. Robot. Autom., 2005, vol. 4, pp. 1986-1991.

[64] W. Zhang, E. Li, J. Xi, J. Chicharo, and X. Dong, "Novel temperatureindependent FBG-type force sensor," Meas. Sci. Technol., vol. 16, pp. 1600-1604, 2005.

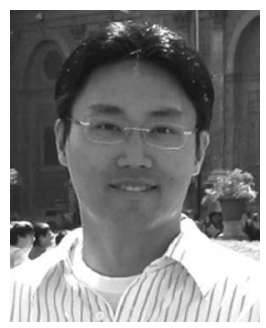

Yong-Lae Park (S'07) received the B.S. degree in industrial engineering in 2000 from Korea University, Seoul, Korea, and the M.S. degree in mechanical engineering in 2005 from Stanford University, Stanford, $\mathrm{CA}$, where he is currently working toward the Ph.D. degree in mechanical engineering.

His current research interests include fiber optic force and tactile sensing of robot manipulators for space and medical applications, the design of haptic master-slave systems for minimally invasive surgery robots, and 3-D smart-robot-structure development.

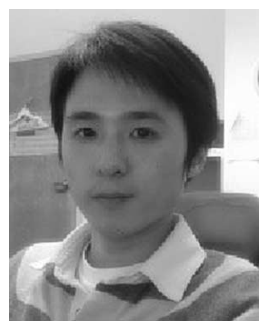

Seok Chang Ryu received the B.S. degree in mechanical engineering in 2002 from Pohang University of Science and Technology, Pohang, Korea, and the M.S. degree in mechanical engineering in 2007 from Stanford University, Stanford, CA, where he is currently working toward the Ph.D. degree in mechanical engineering.

His was with Robostar Corporation, Ltd., Seoul, Korea, where he was engaged in the development of trajectory planners for the Selective Compliant Assembly Robot Arm and wafer-transfer robots. His current research interest include medical application of robots.

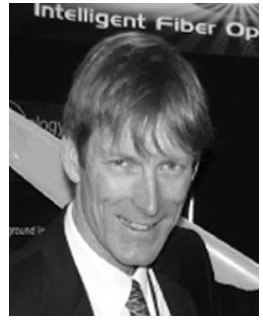

Richard J. Black (M'82) received the B.Sc. (Hons.) degree in physics from the University of Canterbury, Canterbury, New Zealand, and the Ph.D. degree in fiber optics from the Research School of Physical Sciences, Australian National University, Canberra, A.C.T., Australia.

$\mathrm{He}$ is a Founding Member and the Chief Scientist with Intelligent Fiber Optic Systems Corporation, Santa Clara, CA, and the Founder of OptoSapiens Design. His current research interest include optical fiber sensing systems with application to structural health monitoring, robotics, and medical devices.

Dr. Black is a member of the Association for Advancement of Artificial Intelligence, the Association for Computing Machinery, the ASM InternationalThe Materials Information Society, the Optical Society of America, the International Society for Optical Engineers, and the Society for the Advancement of Material and Process Engineering. 


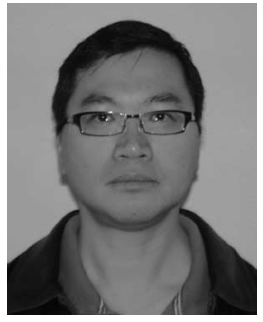

Kelvin K. Chau (M'85) received the B.S. degree in engineering physics/optics from the University of California, San Diego, in 1985 and the M.S. degree in electrical engineering from San Jose State University, San Jose, CA, in 1990.

$\mathrm{He}$ has been engaged in complex optoelectronic system integration and product development. He is currently with Glimmerglass Networks, Hayward, $\mathrm{CA}$, where he is involved in the development of highport-count 3-D optical microelectromechanical system switches for commercial applications.

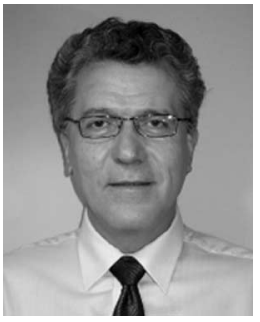

Behzad Moslehi (M'84-SM'98) received the B.S. degree in electrical engineering in 1978 from AryaMehr University of Technology, Tehran, Iran, and the M.S. degree in electrical engineering in 1980, the M.S. degree in applied physics, and the Ph.D. degree in electrical engineering in 1984 from Stanford University, Stanford, CA.

His current research interests include photonic signal processing, sensing, communications, and networking for applications in avionics, safety, life sciences, and energy. He is the Founder and Chief Executive Officer/Chief Technology Officer of Intelligent Fiber Optic Systems Corporation, Santa Clara, CA.

Dr. Moslehi is a member of the Optical Society of America, the International Society for Optical Engineers, the Society for the Advancement of Material and Process Engineering, the Society of Petroleum Engineers, the American Wind Energy Association, and Sigma Xi.

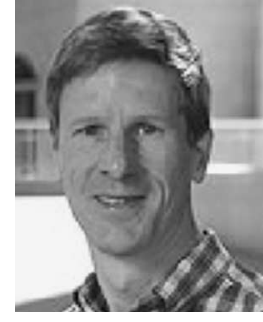

Mark R. Cutkosky (M'93) received the Ph.D. degree in mechanical engineering from Carnegie Mellon University, Pittsburgh, PA, in 1985.

$\mathrm{He}$ is currently a Professor of mechanical engineering with Stanford University, Stanford, CA. His current research interests include robotic manipulation and tactile sensing and the design and fabrication of biologically inspired robots.

Prof. Cutkosky received a Fulbright Faculty Chair, the Charles M. Pigott Professorship, and National Science Foundation Presidential Young Investigator Award. He is a member of the American Society of Mechanical Engineers and Sigma Xi. 